\title{
Guts of surfaces in punctured-torus bundles*
}

\author{
Thilo Kuessner \\ Universität Siegen, Mathematisches Institut, Flexstr.3, 57068 Siegen, \\ kuessner@mathuni-siegen.de
}

\begin{abstract}
Let $M$ be a hyperbolic manifold of finite volume which fibers over the circle with fiber a once punctured torus, and let $S$ be an arbitrary incompressible surface in $M$. We determine the characteristic Jaco-Shalen-Johannsonsubmanifold of $M-S$ and show, in particular, that $\operatorname{Guts}(M, S)$ is empty.
\end{abstract}

\section{Introduction}

Hyperbolic volume is an important homotopy invariant of hyperbolic manifolds and it is an interesting question to relate it to other topological invariants of (special classes of) hyperbolic 3-manifolds.

There is a topological definition of hyperbolic volume, due to Gromov([5]). He defined a homotopy invariant $\|M\|$, the simplicial volume, and proved:

$$
\|M\|=\frac{1}{V_{n}} \operatorname{Vol}(M)
$$

if $M$ is an n-dimensional hyperbolic manifold of finite volume. ( $V_{n}$ denotes the volume of a regular ideal straight n-simplex in hyperbolic n-space.)

There is an obvious inequality

$$
\|M\| \geq \frac{1}{n+1}\|\partial M\|
$$

for any compact n-manifold, due to the fact that the boundary operator has norm $\leq n+1$. If $M$ is a hyperbolic 3-manifold of finite volume, then $\|\partial M\|=0$, so this inequality gives no information.

Let $M$ be a hyperbolic manifold of finite volume and $S$ an arbitrary incompressible surface in $M$. Agol used the simplicial volume to prove the inequality

$$
\operatorname{Vol}(M) \geq-2 V_{3} \chi(\operatorname{Guts}(M, S)) .
$$

${ }^{*}$ Mathematics Subject Classification:57M27 
(We will explain the guts-terminology in the preliminaries, section 2.) In particular, if $M_{S}:=M-\mathcal{N}(S)$ (that is, $M$ cut along $S$ ) admits a hyperbolic metric with totally geodesic boundary $\partial_{1} M_{S}:=S_{1} \cup S_{2}$ (where $S_{1}, S_{2}$ are the two copies of $S$ in $\left.\partial M_{S}\right)$ and cusps corresponding to $\partial_{0} M_{S}:=\partial M_{S}-\partial_{1} M_{S}$, then

$$
\|M\| \geq-\chi(S)=\frac{1}{4}\left\|\partial_{1} M_{S}\right\| .
$$

Agol used his inequality in [2] to get useful estimates for the hyperbolic volume of 2-bridge links, and he suggested (in section 10) that the inequality should also be applied to other classes of 3-manifolds, where incompressible surfaces are wellunderstood, namely alternating link complements and punctured-torus bundles.

For alternating link complements this task has been done in [10]. Lackenby showed the fairly surprising theorem that the hyperbolic volume of an alternating link complement is coarsely proportional to the twist number $t$ of the alternating diagram, precisely $\frac{1}{2} V_{3}(t-2) \leq \operatorname{Vol}(M) \leq 10 V_{3}(t-1)$. (The lower bound is exactly $-V_{3}(\chi(G u t s(M, B))+\chi(G u t s(M, W)))$, where $B$ and $W$ are the checkerboard surfaces of a twist-reduced, prime, alternating link diagram. The upper bound is obtained by Agol and D.Thurston using ad-hoc methods.)

In this paper we determine the guts for an arbitrary incompressible surface in a hyperbolic once-punctured-torus bundle.

Theorem 1 : Let $M$ be a once-punctured-torus bundle over $\mathbb{S}^{1}$ with hyperbolic monodromy. Let $S$ be an arbitrary connected, two-sided, properly embedded, incompressible surface in $M$, not isotopic to the fiber nor the boundary torus. Associated to $(M, S)$ there is a natural number $k$ such that the following holds:

(i) if $k$ is even, then $k=-\chi(S)$ and the components of the characteristic submanifold of $M_{S}$ are $k$ regular neighborhoods of essential squares and the boundary annuli $\partial M_{S} \cap \partial M$, and $k$ Seifert fibered solid tori,

(ii) if $k$ is odd, then $2 k=-\chi(S)$ and the components of the characteristic submanifold of $M_{S}$ are $k$ regular neighborhoods of essential squares and the boundary annuli $\partial M_{S} \cap \partial M, k$ Seifert fibered solid tori, and one handlebody which is an $I$-bundle over a one-sided, properly embedded surface.

In both cases, Guts $(M, S)$ is empty.

The proof, which is given in section 3, proceeds by an elementary analysis of the incompressible surfaces given by the classification due to Floyd-Hatcher and Culler-Jaco-Rubinstein.

Theorem 1 is, of course, disappointing from the point of view of Agol's inequality. It says that Agol's inequality can not provide nontrivial estimates for the hyperbolic volume of once-punctured-torus bundles. 
We mention that, on the other hand, we have an upper bound for the hyperbolic volume in terms of the monodromy. We denote by $t_{l}$ the Dehn-twist in the longitude and by $t_{m}$ the Dehn-twist in the meridian of $T$, the once-punctured torus. It follows from Murasugi's theorem ([3], prop.1.3.1) that the mapping tori with monodromy $A$ and $B$ are homeomorphic if and only if $A$ is conjugate to $B^{ \pm 1}$. Moreover, it is well-known that any mapping class of the once punctured torus is conjugate to one of the form $A= \pm t_{l}^{l_{1}} t_{m}^{m_{1}} \ldots t_{l}^{l_{r}} t_{m}^{m_{r}}, l_{1}, \ldots, l_{r}, m_{1} \ldots, m_{r} \in \mathbb{N}$. (That one can get $l_{1}, \ldots, m_{r} \geq 1$ after suitable conjugation follows from the argument on page 267 of [4].)

Theorem 2 : Let $M$ be a once-punctured-torus bundle over $\mathbb{S}^{1}$ with hyperbolic monodromy $A=t_{l}^{l_{1}} t_{m}^{m_{1}} \ldots t_{l}^{l_{r}} t_{m}^{m_{r}}$. Assume that $l_{1}, \ldots, l_{r}, m_{1}, \ldots, m_{r} \geq 1$. Then

$$
\operatorname{Vol}(M) \leq V_{3} \sum_{i=1}^{r} l_{i}+m_{i} .
$$

Proof: $\quad$ Let $A \in S L(2, \mathbb{Z})$ be the monodromy of the bundle, and let $l$ be a minimal $A$-invariant path in the dual tree to the Farey-triangulation. (All notions are explained in section 2.3. or in [4].) Associated to $l$ there is an ideal triangulation, see the appendix of [4]. Parker proved in [13] that these ideal triangulation can be realized as a triangulation by straight ideal simplices (cf. also [9] for a stronger statement). It is well-known that any straight ideal 3-simplex in hyperbolic 3space has volume $\leq V_{3}$ (cf. [11]). Hence $\operatorname{Vol}(M) \leq k V_{3}$, for the number $k$ of tetrahedra in the ideal triangulation.

The tetrahedra in the ideal triangulation correspond precisely to the vertices of a minimal $A$-invariant path in the dual tree to the Farey triangulation. Each minimal $A$-invariant path belongs to the strip drawn in [4], figure 3 . It is clear from [4], figure 3, that the number of vertices (modulo the action of $A$ ) is $l_{1}+m_{1}+\ldots+l_{r}+m_{r}$. This implies the claimed inequality.

Equality in the inequality of Theorem 2 holds for powers of the Anosov cat map (i.e., finite covers of the figure-eight knot complement).

We emphasize that Theorem 2 is not true without the assumptions $l_{1}, \ldots, l_{r}, m_{1}, \ldots, m_{r} \geq 1$. 


\section{Preliminaries}

\subsection{3-manifolds with boundary-patterns}

We recall some terminology from [8]. Let $N$ be a compact $n$-manifold. A boundary-pattern for $N$ consists of a set $\underline{n}$ of compact, connected $(n-1)$-manifolds in $\partial N$, such that the intersection of any $i$ of them is a (possibly empty) $(n-i)$ manifold.

A map $f:(M, \underline{m}) \rightarrow(N, \underline{n})$ is called admissible if

$$
\underline{m}=\bigcup_{G \in \underline{n}}\left\{\text { components of } f^{-1} G\right\} .
$$

An admissible homotopy is an admissible map $f:(M \times I, \underline{m} \times I) \rightarrow(N, \underline{n})$, where $\underline{m} \times I=\{G \times I: G \in \underline{m}\}$.

Let $(I, \underline{i})$ be the closed intervall with the full boundary-pattern (i.e., consisting of both endpoints). An admissible curve in $N$ is an admissible mapping $k:(I, \underline{i}) \rightarrow$ $(N, \underline{n})$ or $k:\left(S^{1}, \emptyset\right) \rightarrow(N, \underline{n})$. A $\partial$-compression disk for $k$ is an admissible mapping $g:(D, \underline{d}) \rightarrow(N, \underline{n})$, where $D$ is a 2-disk with $j$ boundary faces, such that $k$ is one of the boundary faces and that the other $j-1$ boundary faces (apart from $k$ ) belong to $\underline{d}$, the boundary pattern of $D$. (The case $j=0$ is admitted if $k:\left(S^{1}, \emptyset\right) \rightarrow(N, \underline{n})$. In this case, $k$ is the only boundary face of $D$.) The curve $k$ is called essential if no compression disk exists. A submanifold $(F, \underline{f}) \subset(N, \underline{n})$ is essential if essential curves in $F$ are also essential in $N$.

A boundary pattern $\underline{n}$ of a 3 -manifold $N$ is called useful if the boundary curve of any admissibly embedded 2-disk $(D, \underline{d})$, where $\partial D$ has $i \leq 3$ faces and $\partial D=\cup\{d: d \in \underline{d}\}$, bounds a disk $D^{\prime}$ in $\partial N$ such that $D^{\prime} \cap\left(\cup_{G \in \underline{n}} \partial G\right)$ is the cone on $\partial D^{\prime} \cap\left(\cup_{G \in \underline{n}} \partial G\right)$.

In what follows, an annulus is an annulus with the full boundary-pattern (i.e., consisting of two circles) and a square is a 2-disk with four boundary faces such that the boundary-pattern consists of two non-adjacent boundary faces.

When talking about Seifert fibrations or $I$-bundles $p: W \rightarrow F$, we will always assume that the boundary pattern $\underline{w}$ of $W$ is such that

$$
\underline{w}=\left\{G ; G=p^{-1} k \text { for some } k \in \underline{f} \text { or } G \text { a component of } \overline{\partial M-p^{-1} \partial F}\right\}
$$

for some boundary pattern $\underline{f}$ of $F$. An admissible $\mathbf{F}$-manifold in $(N, \underline{n})$ is a submanifold $W \subset N$ such that each component of $W$ is an admissibly embedded $I$-bundle or Seifert fibre space and that $\overline{\partial W \backslash \partial N}$ is essential. (In particular, $\overline{\partial W \backslash \partial N}$ consists of essential squares, annuli and tori.)

Example: Assume $M$ fibers over $\mathbb{S}^{1}$ with fiber $F$ and $(N, \underline{n})=\left(M_{F}, F_{1} \cup F_{2}\right)$. Then $(N, \underline{n})$ is an $I$-bundle over $F$. Indeed, $\underline{n}$ meets the above definition for the boundary pattern $\underline{f}=\emptyset$ of $F$. 
An admissible F-manifold $W \subset N$ is called characteristic if for every component $V$ of $\overline{M \backslash W}, W \cup V$ is not an admissible F-manifold, and if every admissible F-manifold in $N$ can be admissibly isotoped into $W$.

Remark: Assume that the complement $\partial_{0} N$ of the boundary pattern in $\partial N$ consists of annuli. The regular neighborhood of any essential square $D$ can be considered as an I-bundle (respecting boundary pattern) by projecting to the product neighborhood of one of those faces of $D$ which belong to the boundary pattern of $D$. (This neighborhood is the base surface of the $I$-bundle, it has empty boundary pattern.) We remark that this is not a maximal $I$-bundle. Indeed, the $I$-bundle projection can be extended (respecting boundary pattern) in the obvious way to a product neighborhood of those annuli in $\partial_{0} N$ which intersect $D$. These larger $I$-bundles have annuli (not squares) as their boundary components. Since this can be done for any essential square, one sees that the boundary of the characteristic submanifold consists of essential tori and annuli only.

The following is Proposition 9.4. and Corollary 10.9. in [8], see also [7].

Proposition 1 (Jaco-Shalen, Johannson): Let $(N, \underline{n})$ be an irreducible, boundaryirreducible 3-manifold with useful boundary pattern. Then there exists a characteristic submanifold in $(N, \underline{n})$, unique up to admissible isotopy.

\subsection{Guts of surfaces}

Let $M$ be a compact 3-manifold (with boundary) and $S$ a two-sided properly embedded surface in $M$. Let $N=M_{S}:=\overline{M-\mathcal{N}(S)}$ be the manifold obtained by splitting $M$ along $S$, that is, the closure of the complement of a regular neighborhood $\mathcal{N}(S)$ of $S$ in $M$.

$\partial N$ contains two copies of $S$, which we denote $S_{1}$ and $S_{2}$. We consider the boundary pattern $\underline{n}=S_{1} \cup S_{2}$. If $S$ was incompressible, then $\underline{n}$ is obviously a useful boundary-pattern.

Definition 1 Let $M$ be a compact 3-manifold (with boundary) and $S$ a two-sided, properly embedded surface in $M$. Assume that $(N, \underline{n}):=\left(M_{S}, S_{1} \cup S_{2}\right)$ satisfies the assumptions of Proposition 1. Then we define Guts $(M, S)$ to be the closure of the complement of the characteristic submanifold of $(N, \underline{n})$ in $N$.

If $M$ is irreducible and boundary-irreducible, and $S$ incompressible, then it can be deduced from Thurston's geometrization theorem for Haken manifolds that $\operatorname{Guts}(M, S)$ admits a hyperbolic metric with $\partial G u t s(M, S) \cap\left(S_{1} \cup S_{2}\right)$ totally geodesic. 


\subsection{Incompressible surfaces in hyperbolic once-punctured-torus bundles}

It is well-known that the mapping class group of the once punctured torus is $S L(2, \mathbb{Z})$, representatives acting linearly on $T^{2}-0=\left(\mathbb{R}^{2}-\mathbb{Z}^{2}\right) / \mathbb{Z}^{2}$. Hence, a oncepunctured-torus bundle $M$ is determined by its monodromy $A \in S L(2, \mathbb{Z}) . M$ is hyperbolic if and only if $A$ is hyperbolic (i.e., diagonalizable and not \pm identity).

Definition 2 : Let $T=T^{2}-D^{2}$ be the once punctured torus and $I=[a, b]$ a closed intervall. A standard saddle of bottom slope $\frac{p}{q}$ and top slope $\frac{r}{s}$ in $T \times I$ is an embedded 8-gon in $T \times I$ with faces (in consecutive order) $e_{1}, \ldots, e_{8}$ such that

- $e_{1}$ and $e_{5}$ are arcs of slope $\frac{p}{q}$ in $T \times\{a\}$,

- $e_{3}$ and $e_{7}$ are arcs of slope $\frac{q}{s}$ in $T \times\{b\}$,

- for $i=2,4,6,8$ is $e_{i}$ of the form $\left\{x_{i}\right\} \times I$ for some $x_{i} \in \partial T$.

Incompressible surfaces in once-punctured-torus bundles have been classified in [3] and (for hyperbolic $M$ ) in [4]. We use the approach of [4].

Consider the ideal Farey-triangulation of the upper half-plane model of the hyperbolic plane $\mathbb{H}^{2}$, which is as follows: the ideal vertices are $\mathbb{Q}$ and $\infty=\frac{1}{0}$, two ideal vertices $\frac{a}{b}$ and $\frac{c}{d}$ are connected by an edge if $a d-b c= \pm 1$.

$S L(2, \mathbb{Z})$ acts on $\mathbb{Q} \cup\{\infty\}$ by fractional linear transformations, this action extends to an action preserving the Farey-triangulation. Let $A \in S L(2, \mathbb{Z})$. Consider a minimal $A$-invariant edge-path $\gamma$ in the Farey-triangulation. Let $\ldots, \frac{a_{-1}}{b_{-1}}, \frac{a_{0}}{b_{0}}, \frac{a_{1}}{b_{1}}, \ldots$ be the vertices of $\gamma$. Since $\gamma$ is $A$-invariant, we have some $k \in \mathbb{N}$ such that $A\left(\frac{a_{i}}{b_{i}}\right)=\frac{a_{i+k}}{b_{i+k}}$ for all $i$.

Let $M$ be the once-punctured-torus bundle with monodromy $A$. To each minimal $A$-invariant edge-path $\gamma$ we associate an incompressible surface $S_{\gamma} \subset M$ as follows.

Consider $M$ as the quotient of $T \times[0,1]$ under the identification $(x, 0) \sim$ $(A(x), 1)$. We consider the surface in $T \times[0,1]$ whose intersection with $T \times$ $\left[\frac{i}{k}, \frac{i+1}{k}\right]$ is the standard saddle with bottom slope $\frac{a_{i}}{b_{i}}$ and top slope $\frac{a_{i+1}}{b_{i+1}}$, for $i=0,1, \ldots, k-1$. Since $A\left(\frac{a_{0}}{b_{0}}\right)=\frac{a_{k}}{b_{k}}$, this gives a properly embedded surface in $M$. By [4], $S$ is incompressible in $M$. If $k$ is even, then $S$ is two-sided and we let $S_{\gamma}:=S$. If $k$ is odd, then $S$ is one-sided and we let $S_{\gamma}$ be the boundary of a regular neighborhood of $S$.

The main result of [4] is that each incompressible two-sided surface in $M$ (apart from the fiber and the boundary torus) arises this way. 


\section{Proof of Theorem 1}

Let $M$ be the once-punctured-torus bundle with hyperbolic monodromy $A$. Let $S_{\gamma}$ be the incompressible two-sided surface associated to the $A$-invariant minimal edge-path $\gamma$, as explained in section 2.3. We distinguish the cases that $k$, the period of $\gamma$, is even or odd.

Case 1: $k$ even.

That is, if $\gamma$ has vertices $\left\{\frac{a_{i}}{b_{i}}: i \in \mathbb{Z}\right\}$ with $A\left(\frac{a_{i}}{b_{i}}\right)=\frac{a_{i+k}}{b_{i+k}}$ for a fixed $k \in \mathbb{N}$ and all $i \in \mathbb{Z}$, then $S_{\gamma}$ is composed of the $k$ standard saddles with bottom slopes $\frac{a_{i}}{b_{i}}$ and top slopes $\frac{a_{i+1}}{b_{i+1}}$, for $i=0,1, \ldots, k-1$. Note that $\chi\left(S_{\gamma}\right)=-k$.

Let $N=M_{S}$. A family of squares in $N$ is constructed as follows. Consider $M$ as a quotient of $T \times[0,1]$ in the obvious way. For each $i=0,1, \ldots, k-1$ let $D_{i}$ be the square embedded in $T \times\left\{\frac{i}{k}\right\}$ such that two non-adjacent boundary faces are arcs of slope $\frac{a_{i}}{b_{i}}$ and the two other non-adjacent boundary faces belong to $\partial T \times\left\{\frac{i}{k}\right\}$. These squares in $M$ give squares in the manifold $M_{S}$ with boundarypattern $S_{1} \cup S_{2}$ (see section 2.2.).

Note that the squares $D_{1}, \ldots, D_{k}$ are essential, because the boundary faces not belonging to the boundary-pattern are essential in $T$ and thus (since $T$ is essential in $M$ ) also in $M$ and $M_{S}$. Thus $D_{1} \cup \ldots \cup D_{k}$ can be isotoped into the characteristic submanifold. Let $V$ be a regular neighborhood of $D_{1} \cup \ldots \cup D_{k}$. (We may, as described in the remark in section 2.1., enlargen each component of $V$ as an I-bundle by a neighborhood of the boundary annulus. This does not change neither the homeomorphism type of the complement nor its boundary pattern.)

Claim: The complement of $V$ consists of $k$ solid tori.

This should be fairly clear from the picture of the standard saddles. To make a precise argument, consider the once-punctured torus $T$ as pictured below, with the pairs of $m$ 's resp. l's to be identified. The standard saddle $K$ with bottom slope $\frac{0}{1}$ and top slope $\frac{1}{0}$ is embedded in $T \times\left[\frac{i}{k}, \frac{i+1}{k}\right]$ as the 8-gon with edges $l_{-} \times\left\{\frac{i}{k}\right\},\left\{p_{1}\right\} \times\left[\frac{i}{k}, \frac{i+1}{k}\right], m_{+} \times\left\{\frac{i+1}{k}\right\},\left\{p_{2}\right\} \times\left[\frac{i}{k}, \frac{i+1}{k}\right], l_{+} \times\left\{\frac{i}{k}\right\},\left\{p_{3}\right\} \times$ $\left[\frac{i}{k}, \frac{i+1}{k}\right], m_{-} \times\left\{\frac{i+1}{k}\right\},\left\{p_{4}\right\} \times\left[\frac{i}{k}, \frac{i+1}{k}\right]$. Let $D$ be the disk, containing $m \times\left\{\frac{i+1}{k}\right\}$, which has $m_{-} \times\left\{\frac{i+1}{k}\right\}$ and $m_{+} \times\left\{\frac{i+1}{k}\right\}$ as two of its boundary faces, and its other two boundary faces on $\partial T \times\left\{\frac{i+1}{k}\right\}$. Then $K \cup D$ decomposes $T \times\left[\frac{i}{k}, \frac{i+1}{k}\right]$ into two connected components. Both are solid tori, indeed one of them is obtained from a cube by identifying two copies of $m \times\left[\frac{i}{k}, \frac{i+1}{k}\right]$, the other is obtained from 
a cube by identifying two copies of $l \times\left[\frac{i}{k}, \frac{i+1}{k}\right]$.

Now, apply

$$
f:=\left(\begin{array}{ll}
a_{i} & a_{i+1} \\
b_{i} & b_{i+1}
\end{array}\right) \in S L(2, \mathbb{Z})
$$

to (the first factor of) $T \times\left[\frac{i}{k}, \frac{i+1}{k}\right]$. Then $K$ is mapped to the standard saddle of bottom slope $\frac{b_{i}}{a_{i}}$ and top slope $\frac{b_{i+1}}{a_{i+1}}$ and $D$ is mapped to $D_{i+1}$. Since $f$ is a homeomorphism, the images of the components of $T \times\left[\frac{i}{k}, \frac{i+1}{k}\right]-(K \cup D)$ are solid tori, i.e., $D_{i+1}$ decomposes the complement of the standard saddle of boundary slopes $\frac{b_{i}}{a_{i}}$ and $\frac{b_{i+1}}{a_{i+1}}$ in $T \times\left[\frac{i}{k}, \frac{i+1}{k}\right]$ into two solid tori. So, if we consider one of the connected components of $M_{S}-V$, e.g. the component bounded by $D_{i}$ and $D_{i+1}$, i.e. intersecting $T \times\left[\frac{i}{k}, \frac{i+1}{k}\right]$ and $T \times\left[\frac{i-1}{k}, \frac{i}{k}\right]$, then this component is obtained from two solid tori (the intersections with $T \times\left[\frac{i}{k}, \frac{i+1}{k}\right]$ resp. $T \times\left[\frac{i-1}{k}, \frac{i}{k}\right]$ ) by glueing them along a al annulus (which is the complement in $T \times\left\{\frac{i}{k}\right\}$ of the disk containing $m \times\left\{\frac{i}{k}\right\}$ bounded by $m_{+} \times\left\{\frac{i}{k}\right\}$, two arcs in $\partial T \times\left\{\frac{i}{k}\right\}$, and $m_{-} \times\left\{\frac{i}{k}\right\}$.) Glueing the two solid tori along the longitudinal annulus yields a new solid torus.

It remains to show that this solid torus is Seifert fibered, respecting the boundary pattern. First remark that, for a solid torus with boundary pattern an annulus, a Seifert fibration in which this boundary pattern consists of boundary fibers exists if and only if this annulus is not meridional, i.e., winds along the core at least once. In our case, the intersection of the boundary pattern with $T \times\left[\frac{i}{k}, \frac{i+1}{k}\right]$ is half of a annulus of slope $\frac{b_{i+1}}{a_{i+1}}$, and the intersection of the boundary pattern with $T \times\left[\frac{i-1}{k}, \frac{i}{k}\right]$ is half of a annulus of slope $\frac{b_{i}}{a_{i}}$. These two half-annuli are glued together to form an annulus. Recall that the core of the solid torus has slope $\frac{b_{i}}{a_{i}}$. Hence the annulus in the boundary pattern is not meridional and there exists a Seifert fibration.

This shows the claim if $k$ was even.

Case 2: $k$ is odd.

In this case, $S$ is constructed as above, but $S_{\gamma}$ is the (connected) boundary of a standard neighborhood $\mathcal{N}(S)$ of $S$. Note that $\chi\left(S_{\gamma}\right)=-2 k$.

The same argument as in case 1 shows that the complement of $\mathcal{N}(S)$ can be cut along $k$ essential squares into $k$ solid tori. So it remains to look at $\mathcal{N}(S)-\mathcal{N}\left(S_{\gamma}\right)=$ $\mathcal{N}(S)-\mathcal{N}(\partial \mathcal{N}(S))$. Now, $S$ is a nonorientable surface of Euler characteristic $-k$ with one boundary component, thus $\mathcal{N}(S)-\mathcal{N}(\partial \mathcal{N}(S))$ is a handlebody which is an $I$-bundle over $S$. 


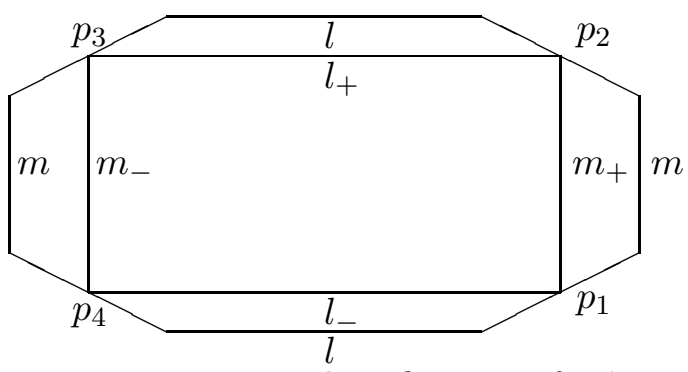

Pairwise identification of $m$ 's and $l$ 's yields punctured torus

Question: It seems somewhat astonishing to me that in all cases considered so far (2-bridge links, alternating links, once-punctured-torus bundles), the characteristic subpairs of $(M, S)$ consist only of regular neighborhoods of essential squares (and boundary annuli) and of Seifert fibered solid tori. The squares arise from apparently different reasons: If $S$ is the spanning surface of a two-bridge link $K \frac{p}{q}$, then the squares correspond to the '2'-s in the associated continued fraction expansion, i.e., to the occurences of $b_{i}=2$ in the expansion $\frac{p}{q}=r+\frac{1}{b_{1}-\frac{1}{b_{2}-\frac{1}{b_{3}-\ldots}}}$.

If $S$ is the black checkerboard surface for an alternating link complement, then the squares correspond (not one-to-one) to the white bigon regions in the alternating diagram. If $S$ is an arbitrary incompressible surface in a once-punctured-torus bundle, then the squares correspond to the vertices of the associated minimal invariant edge-path in the Farey-triangulation.

This naturally raises the question, how general this phenomenon may be among arbitrary hyperbolic 3-manifolds, i.e. whether there is some unified reason restricting the existence of essential annuli in $M-S$ and explaining the occurence of squares.

Higher genus. The analogue of corollary 1 for surface bundles of fiber genus $\geq 2$ is not true. Counterexamples can be found in [6]. Namely, it follows from [6] (the remark after the corollary on p.228), that, for example, the complement of the knot $K_{\frac{4}{11}}$ fibers over the circle. On the other hand, [6] constructs incompressible surfaces $S$ corresponding to the continued fraction expansion $\frac{4}{11}=[3,4]$ and then $\chi(G u t s(M, S))=-1$ follows from the computation in [2], section 7 . This negative result is, of course, positive from the point of view of hyperbolic volume. It says that Agol's inequality provides nontrivial estimates for surface bundles of higher fiber genus. However, to explore this in a systematic way, one would need a classification of incompressible surfaces in surface bundles. 


\section{References}

[1] AGOL (I.). Topology of hyperbolic 3-Manifolds, PhD-Thesis UCSD, (1998), http://www.math.uic.edu/ agol/shortthesis.ps

[2] AGOL (I.). Lower Bounds on volumes of hyperbolic HaKen maniFOLDS, Preprint, http://front.math.ucdavis.edu/math.GT/9906182.

[3] CULlER (M.), JACO (W.) and RUBINSTEIN (H.). - InCOMPRESSiBle SURFACES IN ONCE-PUnCTURED TORUS Bundles, Proc. London Math. Soc., 45, (1982), pp.385-419.

[4] FLOYD (W.) and HATCHER (A.). - INCOMPRESSIBle SURFACES IN Punctured-Torus Bundles, Top. Appl., 13, (1982), pp.263-282.

[5] GROMOV (M.). - Volume and bounded cohomology, Publ. IHES, 56, (1982), pp.5-100.

[6] HATCHER (A.) and THURSTON (W.). - InCOMPRESSible SURFACES IN 2-BRIDGe KNOT COMPLEMEnts, Invent. Math., 79, (1985), pp.225-246.

[7] JACO (W.) and SHALEN (P.). - SEIFERT FIBEREd SPACES IN 3-MANifOldS, Mem. Amer. Math. Soc., 21, (1979), no.220.

[8] JOHANNSON (K.). - Homotopy Equivalences of 3-Manifolds With Boundaries, Lecture Notes in Mathematics 761, Springer Verlag, (1979).

[9] LACKEnBY (M.). - The CANonical Decomposition of OnCEPunctured torus Bundles, Comm. Math. Helv., 78, (2003), pp.363-384.

[10] LACKEnBY (M.). - The volume of Hyperbolic Alternating Link Complements, Proc. London Math. Soc., 88, (2004), pp.204-224.

[11] MilnOR (J.). - - Computation of volume , http://www.msri.org/publications/books/gt3m/PS/7.ps

[12] NEUMANN (W.) and SWARUP (G.). - CANonical DeCompositions of 3-MANIFOlDS, Geom. Topol., 1, (1997), pp.21-40.

[13] PARKER (J.). - Tetrahedral decompositions of Punctured torus BUNDLES, London Math. Soc. Lecture Notes, 299, (2003), pp.275-291. 